\title{
APLICACIÓN DE TÉCNICAS DE APRENDIZAJE AUTOMÁTICO PARA LA CLASIFICACIÓN DE ACTIVIDADES MEDIANTE UNA MULETA INTELIGENTE PARA ESCLEROSIS MÚLTIPLE
}

\begin{abstract}
Resumen
El nivel de actividad física diaria que un paciente de Esclerosis Múltiple es capaz de realizar se ha demostrado que es una importante fuente de información para el seguimiento de la enfermedad y la adaptación individualizada de las terapias. Así, en este trabajo se propone el diseño de un clasificador de actividades de la vida diaria, realizado mediante la combinación de dos técnicas de Inteligencia Artificial (RandomForest y Redes Neuronales Artificiales), el cual podría facilitar información de valor a las y los terapeutas.
\end{abstract}

Sergio Lucas, Asier Brull, Eva Portillo, Asier Zubizarreta, Itziar Cabanes

Departamento de Ingeniería de Sistemas y Automática, Escuela de Ingeniería de Bilbao, Universidad del País Vasco (UPV/EHU)

Palabras clave: Esclerosis Múltiple, clasificador, RandomForest, Redes Neuronales Artificiales

\section{Introducción}

Dentro de las diferentes afecciones neurológicas la Esclerosis Múltiple (EM) es una de las que ocasiona mayor deterioro físico y psíquico. Publicaciones recientes [7] han estimado que en el mundo hay aproximadamente 2.8 millones de personas que padecen EM, de las cuales 700.000 se encuentran en Europa y 50.000 pertenecen a España.

Aproximadamente, el $85 \%$ de los pacientes de EM padecen el tipo evolutivo conocido como Remitente-Recurrente (EMRR) [9], el cual se caracteriza por ataques imprevisibles o brotes. Como consecuencia de uno de estos brotes, pueden quedar secuelas en forma de dificultad a la hora de ejecutar ciertas tareas o funciones. De hecho, al cabo de 15 años un elevado porcentaje de las personas afectadas requiere de elementos de ayuda técnica, como muletas y bastones, para mantener su autonomía [8].

Debido a que la EM es una enfermedad actualmente sin cura, de carácter crónico y que la media de edad se sitúa en 30 años, esta enfermedad ocasiona un gran coste sanitario. Dependiendo del estadio de la enfermedad estos costes pueden variar desde los 20.000 euros anuales hasta los 70.000 en la fase más severa [3]. La inmensa mayoría de estos costes se centran en ralentizar la enfermedad y paliar las secuelas. Una de las soluciones actuales para mejorar la calidad de los pacientes es la rehabilitación, ya que con un par de sesiones semanales supervisadas por expertos se puede incrementar la fuerza y la capacidad física del paciente [2].

Con el objetivo de proporcionar una rehabilitación óptima, es necesario una correcta y actualizada evaluación y caracterización del estado funcional del paciente. Para ello, actualmente se hace uso de escalas clínicas normalizadas o de soluciones invasivas que requieren de un entorno clínico para el diagnóstico. Sin embargo, estas caracterizaciones son puntuales y no tienen en cuenta aspectos relevantes de la enfermedad como la fatiga. Por tanto, la capacidad para definir terapias precisas se ve limitada de manera significativa al no realizarse una monitorización continua del paciente.

Se ha demostrado que el nivel de actividad física diaria que el paciente es capaz de realizar es una importante fuente de información para el seguimiento de la enfermedad y la adaptación individualizada de las terapias [5]. Dentro de las soluciones propuestas para el diagnóstico del estado del paciente EM, destacan aquellas basadas en dispositivos pasivos para el análisis de la marcha $[5]$.

En este trabajo se presenta una combinación de técnicas de Inteligencia Artificial (IA), más concretamente la combinación de un RandomForest con Redes Neuronales Artificiales (ANN), para desarrollar un clasificador capaz de diferenciar entre distintas actividades físicas diarias realizadas, ofreciendo a los terapeutas una forma de conocer el avance y el estado de la enfermedad de forma continua. La información que se va caracterizar es la extraída de una Contera Inteligente (CI) emplazada en una muleta, la cual es capaz de obtener los parámetros más importantes del patrón de marcha de los pacientes EM de forma no invasiva y continua.

El resto del artículo está estructurado de la siguiente forma. En la sección 2, se presenta una breve descripción de la CI. En la sección 3 se define la metodología, comenzando por la obtención de la base de datos experimental, para luego centrarse en el empleo de las dos técnicas de IA. En la 
sección 4 se presentan los resultados y, por último, en la sección 5 se recogen las principales conclusiones del trabajo.

\section{Contera Inteligente}

La CI utilizada para extraer los datos del patrón de marcha es una contera diseñada y desarrollada por el grupo de investigación Sensorización Virtual para Bioingeniería (UPV/EHU) [1], el cual lleva trabajando desde el año 2017 en la monitorización continua y no invasiva del estado del paciente EM.

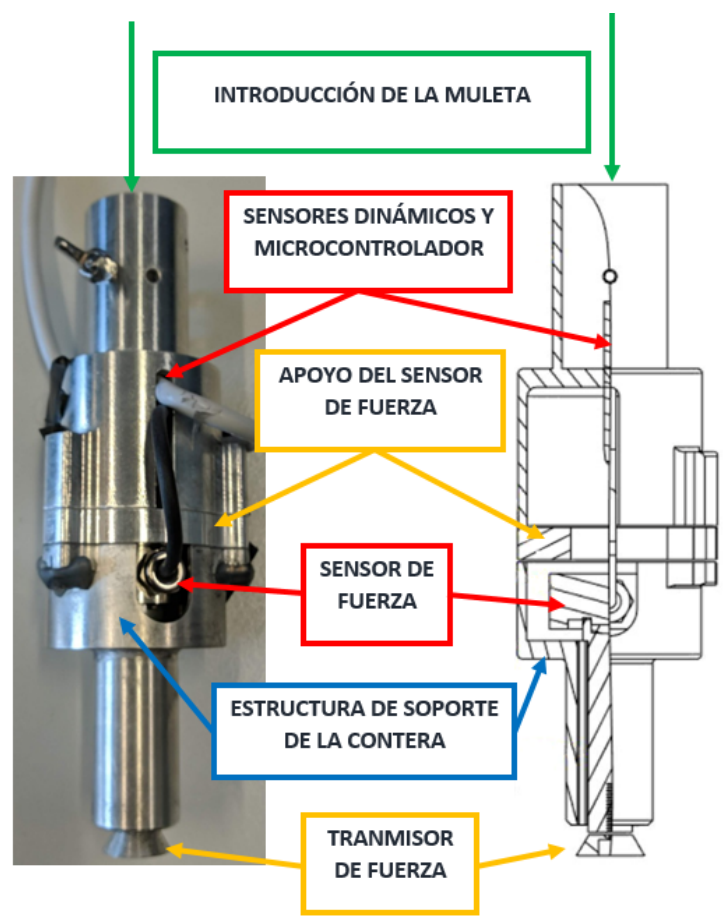

Figura 1: Distribución sensores en la contera

El prototipo de la CI se muestra en la Figura 1. Se trata de una contera fabricada en aluminio que es adaptable a diferentes elementos de ayuda técnica, bastones y muletas.

Debido a los sensores que lleva incorporada la CI [1] es posible capturar las variables básicas necesarias tanto para la caracterización de la marcha como del equilibrio de los pacientes EM [4]. En total se capturan las siguientes variables:

- Velocidad angular en los tres ejes.

- Aceleración lineal en los tres ejes.

- Campo magnético en los tres ejes.

- Ángulos de Euler (Roll, Pitch, Yaw).

- Presión atmosférica.
- Fuerza axial.

El procesamiento de los datos de los sensores se realiza con un circuito integrado BLE nano v2, que permite enviar los datos de forma inalámbrica mediante bluetooth a otro dispositivo con una frecuencia de muestreo de 50 hercios y con un bajo consumo de energía.

\section{Metodología}

\subsection{Base de Datos experimental}

En el caso del presente trabajo, el clasificador va a diferenciar entre cinco actividades que se pueden realizar diariamente: 1) andar recto lento; 2) andar recto rápido; 3) subir escaleras; 4) bajar escaleras; 5) posición estática.

\subsubsection{Metodología experimental}

Para alcanzar un correcto nivel de generalización en la ANN es imprescindible realizar una correcta etapa de entrenamiento. Para ello, se necesita obtener una base de datos experimental significativa y representativa. Así, se define una metodología experimental basada en recrear las actividades que se quieren clasificar mediante cinco pruebas: a) recorrer tres veces una distancia de treinta metros a una velocidad normal para el participante; b) recorrer tres veces la misma distancia, pero con una velocidad $30 \%$ superior; c) subir cuatro veces quince escalones; d) bajar cuatro veces quince escalones; e) mantener una posición estática durante diez segundos. Tanto las repeticiones como la distancia a recorrer se ha definido para lograr una base de datos balanceada.

Debido a que las personas que padecen EM son una población vulnerable y por motivos de la situación sanitaria que se está viviendo derivada de la COVID19, estas pruebas se han realizado con seis personas sanas.

\subsubsection{Creación de features}

Dentro de este campo de investigación es muy común definir una serie de indicadores o features que permitan caracterizar el patrón de marcha. En el caso de estudio, estas features se calculan mediante la aplicación de estadísticos (media, varianza, kurtosis, percentiles, etc.) a cada una de las series temporales obtenidas con la contera por cada participante y por cada prueba. Para calcularlas de forma adecuada, se segmentan las series temporales en paquetes más pequeños de información mediante técnicas de ventana (ver Figura 2). Para las actividades que requieran movimiento (andar, subir y bajar escaleras) se aplican técnicas 


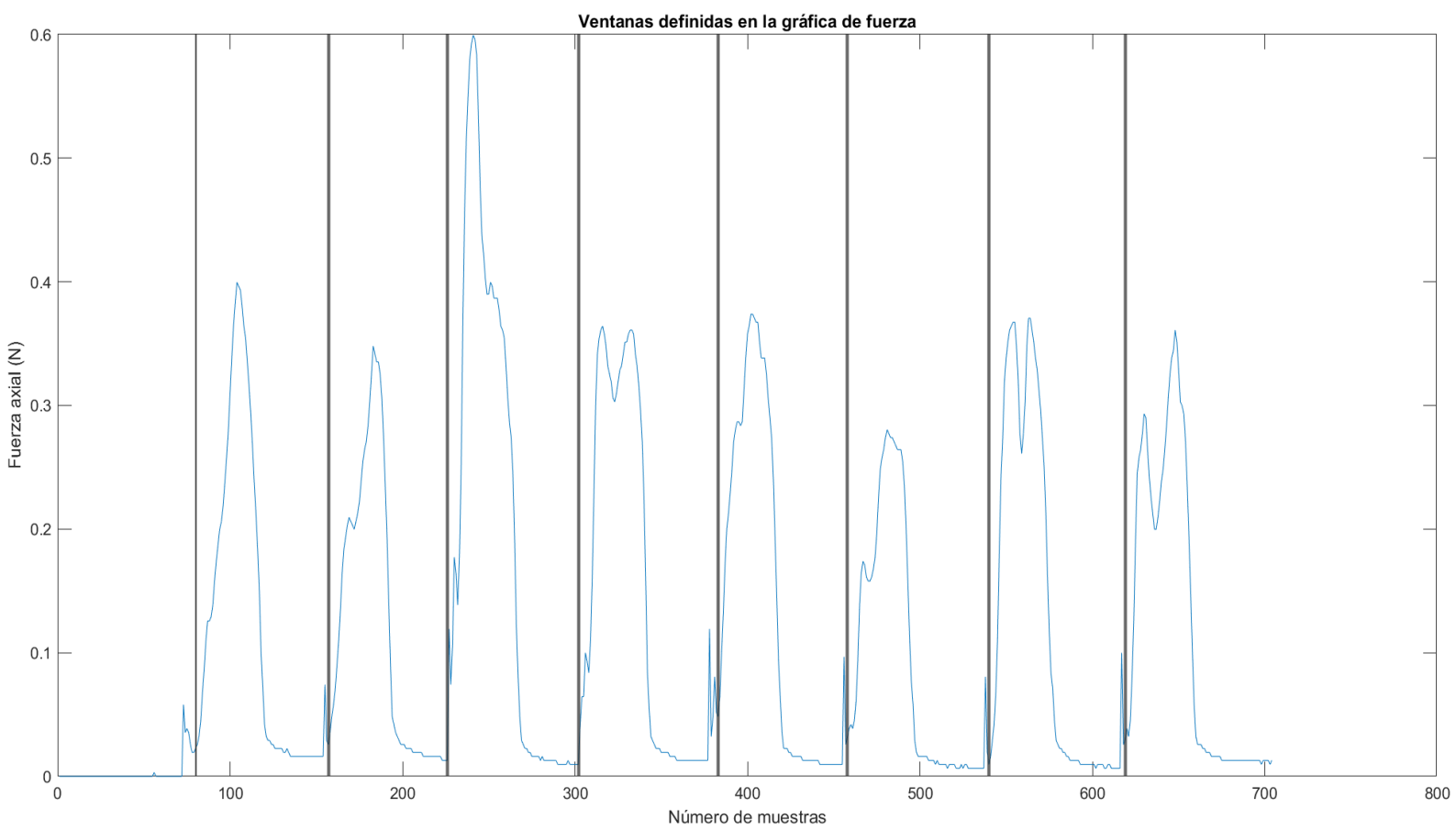

Figura 2: Segmentación variables

de ventana por evento, el cual será el inicio del apoyo en el paso, por tanto, en estos casos los segmentos se corresponderán a cada paso dado por el participante. En el caso de la actividad de mantenerse en estático, la segmentación se realiza mediante ventanas deslizantes. Una vez calculadas todos los segmentos, se aplica a cada uno de ellos los estadísticos elegidos, conformando así las features.

A la base de datos se le debe someter a un proceso de balanceo, de tal forma que, la base de datos final de la que se dispone está formada por 671 muestras o pasos, repartidos tal y como aparece en la Tabla 1, y 177 features por cada muestra. Mencionar que la etiqueta estar quieto se ha introducido en este caso de estudio debido a que el tiempo que los pacientes de EM emplean en sedestación es importante para inferir su estado $y$, por tanto, se considera que el clasificador debe distinguir este tipo de actividad.

\subsection{Reducción de la dimensión mediante RandomForest}

A la ANN se le introducen como variables de entrada las diferentes features calculadas en el paso anterior, sin embargo, se tiene un número excesivo de features (177) en comparación con el número de muestras (671), lo cual podría derivar en un modelo sobre-ajustado. Para solventar este inconveniente, se decide reducir la dimensión de las variables de entrada mediante la estimación de sus importancias relativas, usando para ello una técnica consolidada como el RandomForest (RF). Una vez estimadas las importancias, se escogen las 20 features más importantes como entradas a la ANN (eje X en Figura 3).

El resultado del RF es un factor de gran influencia en el entrenamiento de las ANN, debido a que define qué features son las variables de entrada a la red. Por tanto, la base de datos se divide de forma balanceada mediante la habitual división 70-30 en el conjunto de entrenamiento-validación y el conjunto de test, respectivamente, y sólo se introduce el conjunto entrenamiento-validación en el RF. A su vez el conjunto de entrenamientovalidación tambien se divide mediante la división 70-30 en el conjunto de entrenamiento y conjunto de validación (conjuntos requeridos en la ANN). Se han escogido estos valores para las divisiones ya que se han utilizado con anterioridad en la literatura en problemas de esta índole.

Para la correcta implementación del método RF también se deben tener en cuenta una serie de hiperparámetros, entre los que se destacan los siguientes:

- Número de features para cada árbol= raíz cuadrada del número total de features de la base de datos.

- Tamaño de las muestras para cada árbol = Número total de muestras, N.

- Número de árboles: este hiperparámetro será objeto de estudio, ya que se deberá escoger un 
Tabla 1: Base de datos experimental balanceada

\begin{tabular}{|c|c|c|c|c|c|}
\hline \multirow{2}{*}{ Participantes } & \multicolumn{5}{|c|}{ Muestras } \\
\cline { 2 - 6 } & Andar Lento & Andar Rápido & Subir Escaleras & Bajar Escaleras & Estar Quieto \\
\hline 1 & 24 & 23 & 24 & 24 & 16 \\
\hline 2 & 24 & 23 & 24 & 24 & 15 \\
\hline 3 & 24 & 25 & 24 & 24 & 17 \\
\hline 4 & 24 & 25 & 23 & 24 & 18 \\
\hline 5 & 24 & 23 & 24 & 24 & 14 \\
\hline 6 & 24 & 25 & 23 & 24 & 17 \\
\hline \hline Total etiqueta & 144 & 144 & 142 & 144 & 97 \\
\hline Muestras totales & \multicolumn{5}{|l}{} \\
\hline
\end{tabular}

valor con el cual los resultados se hayan estabilizado y el coste computacional sea asumible. Para ello, se van a valorar diferentes números de árboles y se van a analizar los resultados.

\subsection{Diseño del clasificador mediante Redes Neuronales Artificiales}

Se decide escoger el modelo de Perceptron Multicapa (MLP) entrenado mediante el algoritmo de retropropagación y la técnica de regulación Early Stopping.

El modelo MLP se plantea para que sólo disponga de una capa oculta. Los hiperparámetros que van a ser objeto de estudio son: a) número de neuronas en dicha capa oculta; b)número de features como variables de entrada. En este segundo hiperparámetro se comienza introduciendo sólo la feature más importante a la red $\mathrm{y}$, posteriormente, se añaden de una a una el resto, según su orden de importancia, hasta que haya un máximo de 20 features. Este número máximo de 20 no es una restricción definitiva en el problema, sino que es el número de partida, si en los resultados se observa que la red debe ser más compleja, se aumentará dicho número.

Para la selección de la red a implantar como clasificador se realiza un ajuste grueso y fino tanto del número de neuronas como del número de features. Para lograr una correcta selección se decide utilizar como criterio el Principio de Parsimonía, que establece que en igualdad de condiciones con el enfoque más sencillo se reduce la varianza del modelo, haciendo que la solución ante un nuevo conjunto de datos sea más robusta que la que se obtiene con modelos más complejos. Es decir, con este criterio se premia el poder de generalización de la redes y la reducción del consumo de recursos. Esto es algo positivo a futuro, ya que se tiene intención de empotrar este clasificador en un sistema móvil.

\section{Resultados}

\subsection{Número definitivo de árboles del RF}

Para seleccionar el número de árboles a implementar en el RF y en concordancia con la literatura [6], se define realizar un estudio con 5000, 2500 y 1500 árboles para barrer un amplio espectro del espacio de posible resultados de este hiperparámetro. Los resultados de este primer estudio para las veinte features más importantes se muestran en la Figura 3 .

La Figura 3 muestra que con 1500 árboles existe mayor variabilidad en los resultados que con los obtenidos con 2500 y 5000 , verificándose así que a mayor número de árboles, mayor precisión en los resultados. Para la selección definitiva de este hiperparámetro, se decide analizar para 2500 y 5000 las variaciones que existen entre dos $\mathrm{RF}$ del mismo número de árboles, es decir, se comprueba la robustez de los resultados del RF, ya que para el presente caso de estudio es de suma importancia que los resultados sean robustos para tener garantías en cuanto a las features seleccionadas. Para realizar este estudio se utiliza como métrica el coeficiente tau de Kendall, el cual se suele utilizar para comparar clasificaciones o rankings, adquiriendo un valor de 1 cuando las clasificaciones son iguales.

Tabla 2: Coeficiente de Kendall

\begin{tabular}{|c|c|}
\hline Num.árboles & Coef. Kendall \\
\hline $5000-5000$ & 1 \\
\hline $2500-2500$ & 0.7789 \\
\hline
\end{tabular}

Los resultados de la Tabla 2 muestran que con 5000 árboles los resultados han convergido, sin embargo, en el caso de 2500 existen diferencias en el orden de importancia de las features entre los dos RFs. Por ello, se decide escoger 5000 árboles como número de árboles definitivo. 
Comparación resultados según número de árboles

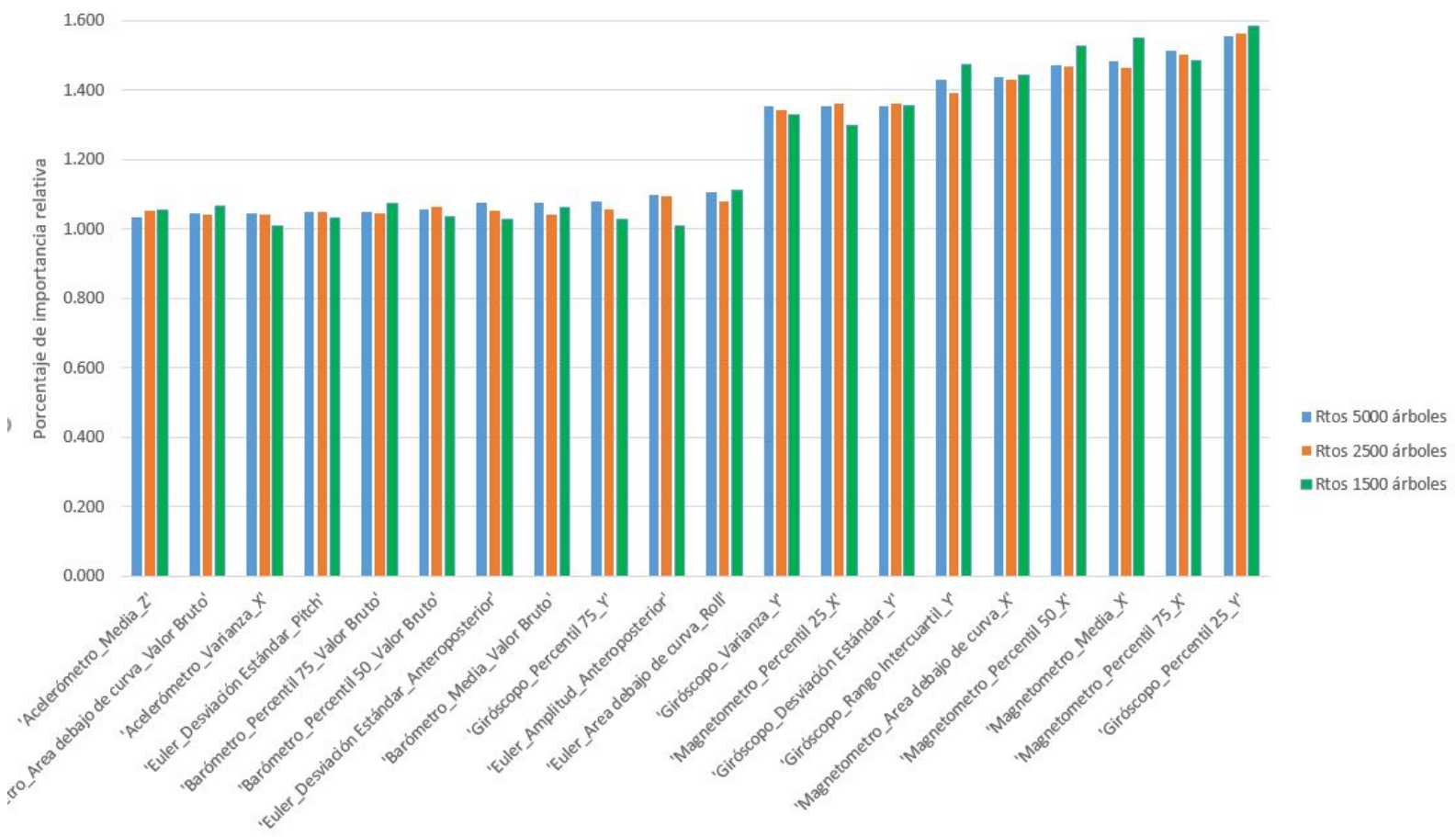

Figura 3: Variación de la importancia relativa con 5000, 2500 y 1500 árboles

\subsection{Redes Neuronales Articiales}

Una vez seleccionado el número de árboles, se realiza un análisis preliminar para comprobar que la metodología planteada es coherente y correcta, es decir, verificar si a partir de las estimaciones calculadas por el RF e introduciendo las features más importantes a la red, se consiguen los mejores porcentajes de acierto en la clasificación. Para ello, se coge una ANN de referencia de 20 neuronas en la capa oculta y se introducen de una en una las feature desde la menos importante hasta la más importante.

En la Figura 4 se puede comprobar cómo el porcentaje de acierto se va incrementando a medida que se van introduciendo features más importantes. Hay casos en los que se puede visualizar como el porcentaje de acierto disminuye. Se ha comprobado que esto se debe a que la feature escogida en ese caso no caracteriza correctamente a una clase en particular, haciendo que el porcentaje de acierto correspondiente a esa clase disminuya drásticamente y con ello el porcentaje global, sin embargo, se tratan de casos aislados. Así, se puede concluir que la metodología planteada es correcta y coherente con los resultados que se esperan.

Las ANN se van a valorar en función del porcentaje de acierto global que presenten. Este porcentaje se calcula con las muestras de test, comparando la salida de la red con la muestra original.
Teniendo esto en cuenta se recuerda que se van a estudiar los siguientes dos hiperparámetros: a) número de neuronas en la capa oculta; b) número de features a introducir en la ANN.

Para barrer un amplio espectro del espacio de posibles resultados se comienza con un ajuste grueso del número de neuronas, definiendo los valores iniciales de 10, 20, 50 y 100. Los resultados que se obtienen con estos número de neuronas son muy similares. Por tanto, se realiza un ajuste fino en torno al número de neuronas de 10, definiendo $1,2,3,4$ y 5 como nuevas opciones. En todos ellos se introducen como máximo 20 features (Figura $5)$.

Se puede diferenciar claramente cómo las redes de 1,2 y 3 neuronas presentan unos porcentajes globales de acierto inferiores que el resto, sin embargo, para el resto de las redes los porcentajes de acierto son muy similares. Además, se observa cómo con un número reducido de features (de 2 a 5 ) se consiguen resultados similares a los obtenidos con redes más complejas (a partir de 10 features).

Para seleccionar la red definitiva a implementar como clasificador se decide testear de nuevo las redes de 4,5 y 10 neuronas con 6 features como máximo, con un nuevo conjunto de test. Este conjunto se forma por las muestras que se habían quedado fuera en la etapa de balanceo. Además, las muestras empleadas en este nuevo grupo de test se tratan de muestras de andar recto lento y 
Porcentaje de acierto ANN 20 neuronas escogiendo features de 1 en 1

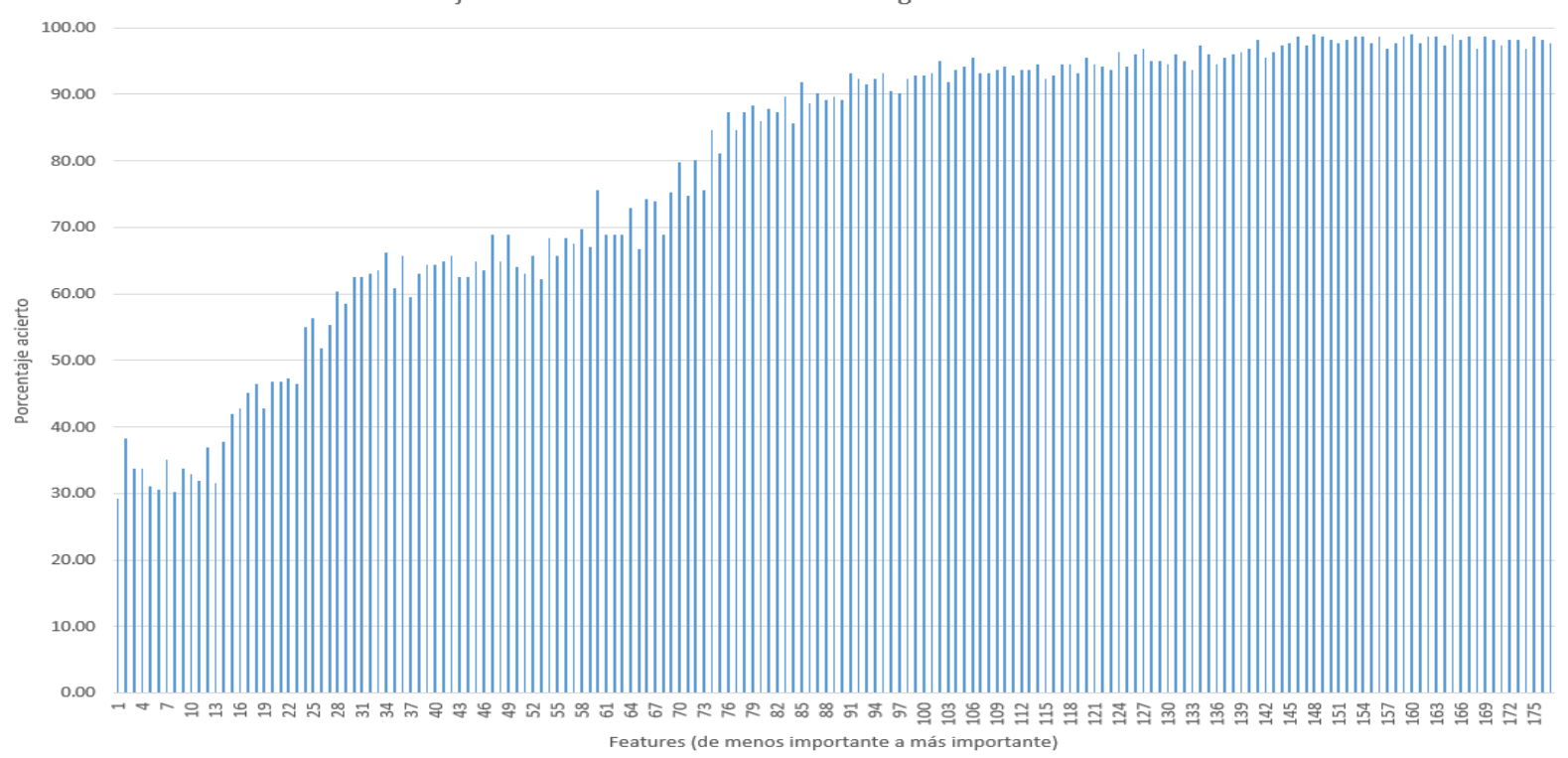

Figura 4: Porcentajes de acierto desde features menos importantes a las más importantes

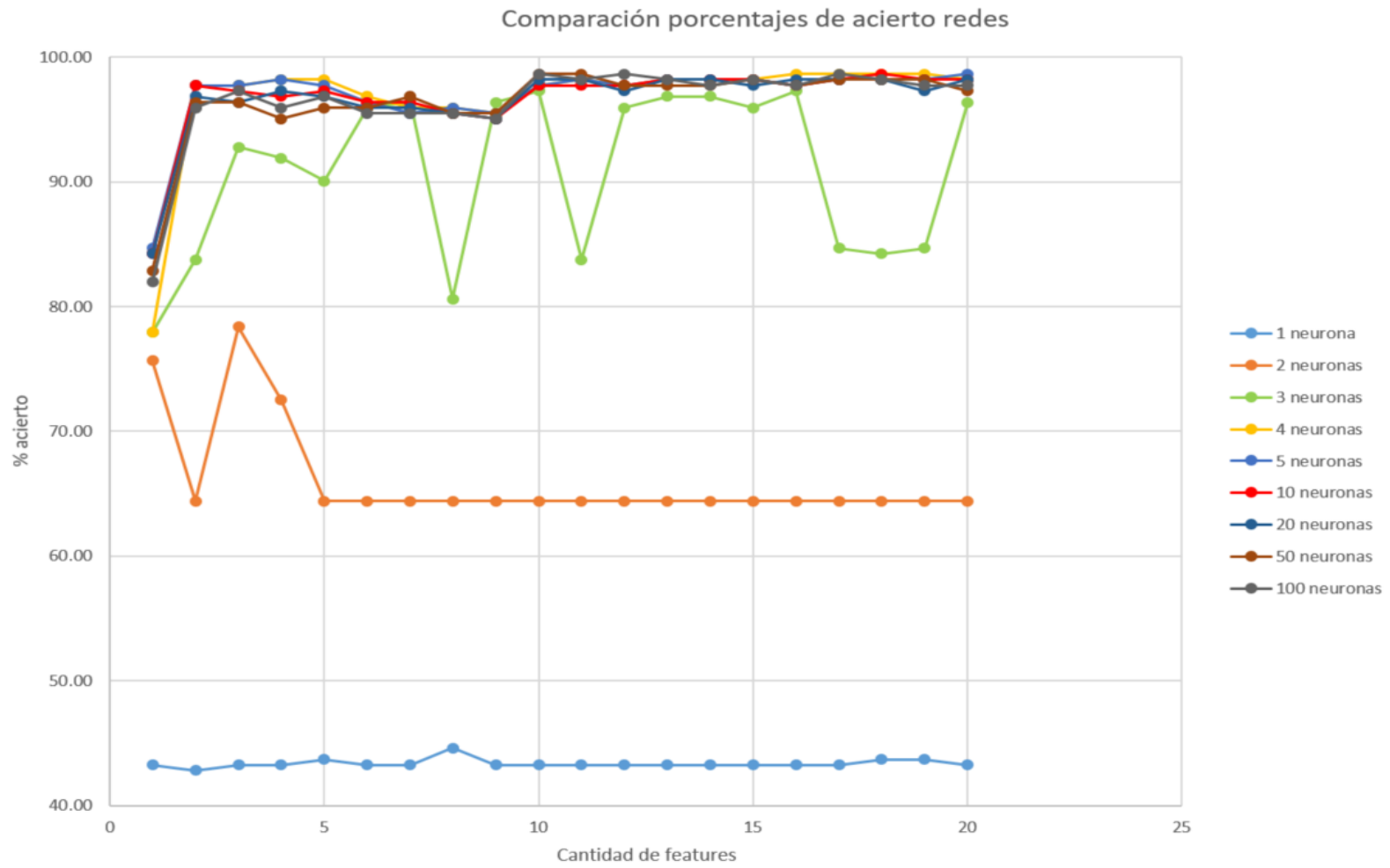

Figura 5: Comparación acierto redes con distintos números de neuronas

Tabla 3: Porcentajes de acierto desglosados red elegida

\begin{tabular}{|c|c|c|c|c|c|c|}
\hline \multirow{2}{*}{$\begin{array}{c}\text { Numero } \\
\text { de } \\
\text { features }\end{array}$} & $\begin{array}{c}\text { Pndar Recto } \\
\text { Lento }\end{array}$ & $\begin{array}{c}\text { Andar Recto } \\
\text { Rápido }\end{array}$ & $\begin{array}{c}\text { Subir } \\
\text { Escaleras }\end{array}$ & $\begin{array}{c}\text { Bajar } \\
\text { Escaleras }\end{array}$ & $\begin{array}{c}\text { Estar } \\
\text { Quiero }\end{array}$ & $\begin{array}{c}\text { Global } \\
\text { De La Red }\end{array}$ \\
\hline 2 & 89.58 & 100.0 & 100.0 & 100.0 & 100.0 & $\mathbf{9 7 . 7 5}$ \\
\hline
\end{tabular}




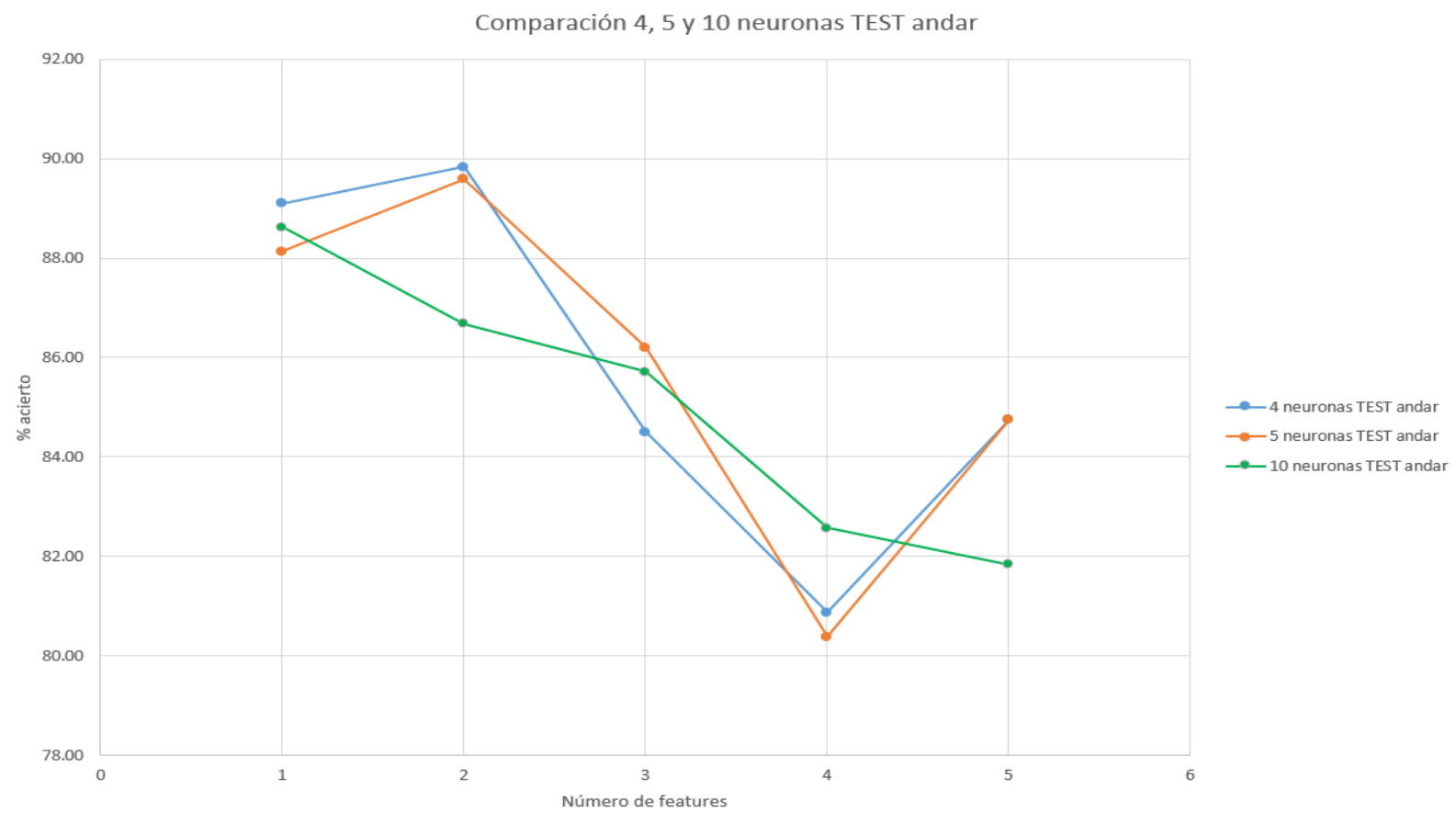

Figura 6: Comparación acierto redes con nuevo conjunto de test

rápido, que se trata de las únicas etiquetas donde falla el clasificador. Los porcentajes de acierto de las redes entrenadas ante este nuevo conjunto de test se muestran en la Figura 6.

Se puede visualizar cómo con 4 neuronas y 2 features se consiguen los mejores resultados. Así, se ha decidido escoger esta red como la red a implantar como clasificador de actividades. Esta red con el conjunto de datos de test inicial presenta los porcentajes descritos en la Tabla 3 .

Para complementar toda la información acerca de este red, a continuación en la Tabla 4 se adjunta la matriz de confusión obtenida.

Tabla 4: Matriz de confusión

\begin{tabular}{|c|c|c|c|c|c|}
\hline Actividades & ARL & ARR & SE & BE & EE \\
\hline ARL & 43 & 5 & 0 & 0 & 0 \\
\hline ARR & 0 & 48 & 0 & 0 & 0 \\
\hline SE & 0 & 0 & 47 & 0 & 0 \\
\hline BE & 0 & 0 & 0 & 47 & 0 \\
\hline EE & 0 & 0 & 0 & 0 & 31 \\
\hline
\end{tabular}

Siendo la primera columna, la etiqueta andar recto lento (ARL); la segunda columna, andar recto rápido (ARR); la tercera, subir escaleras (SE); la cuarta, bajar escaleras (BE); y la quinta, estar estático (EE

En la Tabla 4 se puede ver que los únicos errores que comete el clasificador son las 5 muestras de andar recto lento que las confunde con andar recto rápido, lo cual se puede explicar debido a la incertidumbre en la velocidad de los pasos durante la realización de las pruebas, si bien por otro lado cierta variabilidad en las velocidades resulta deseable para entrenar las redes.

\section{Conclusiones}

La mejora en las monitorizaciones del estado de los pacientes EM es fundamental para que se puedan adecuar las terapias de forma efectiva y personalizada. En este sentido, una monitorización continua y no invasiva del patrón de marcha junto a una clasificación de las actividades de la vida diaria que realizan, podría facilitar información de valor a las y los terapeutas para lograr dicho objetivo.

Así, en este trabajo se propone una combinación de dos técnicas de Inteligencia Artificial, como son el RandomForest y las Redes Neuronales Artificiales, para diseñar un clasificador que permita distinguir entre las actividades andar recto lento, andar recto rápido, subir escaleras, bajar escaleras y estar quieto.

Los resultados que se han conseguido son muy satisfactorios, habiéndose logrado porcentajes de acierto globales con las muestras de test cercanos al 98\%. Por tanto, se puede concluir que la metodología propuesta es coherente y válida con los resultados obtenidos, y se estaría en disposición de validar el funcionamiento del clasificador en personas con patología EM cuando la situación sanitaria lo permita. En este sentido, debido a las diferencias en los patrones de marcha de los pacientes EM, donde existe mayor variabili- 
dad incluso para pacientes con mismas patologías, la estrategia que se abordará será particularizar este clasificador para cada persona, es decir, realizar una estrategia individual y personalizada para cada paciente, siendo la estrategia que se ha utilizado en este artículo el punto de partida.

\section{Agradecimientos}

Este trabajo ha sido financiado por la Universidad del País Vasco UPV/EHU (GIU19/045), FEDER/Ministerio de Ciencia, Innovación y Universidades - Agencia Estatal de Investigación/ DPDPI2017-82694-R y Universidad del País Vasco UPV/EHU (PIF18/067).

\section{English summary}

\section{APPLICATION OF MACHINE LEARNING TECHNIQUES FOR ACTIVITY CLASSIFICATION US- ING AN INTELLIGENT CRUTCH FOR MULTIPLE SCLEROSIS}

\begin{abstract}
The level of daily physical activity that a Multiple Sclerosis patient is able to perform has been shown to be an important source of information in order to monitorise the disease and adapt therapies in a personalised way. Consequently, in this work a novel desing of a daily physical activity classifier is proposed by the combination of two Artificial Intelligence techniques (a RandomForest and Artificial Neural Networks), which can provide an useful information for the therapists.
\end{abstract}

Keywords: Multiple Sclerosis, classifier, RandomForest, Artificial Neural Network.

\section{Referencias}

[1] A. Brull, A. Zubizarreta, I. Cabanes, and A. Rodriguez-Larrad. Sensorized tip for monitoring people with multiple sclerosis that require assistive devices for walking. Sensors (Switzerland), 20(15):1-20, 2020.

[2] P. Flachenecker. Clinical implications of neuroplasticity - the role of rehabilitation in multiple sclerosis. Frontiers in Neurology, 6(MAR):1-4, 2015.
[3] G. Kobelt, A. Thompson, J. Berg, M. Gannedahl, and J. Eriksson. New insights into the burden and costs of multiple sclerosis in Europe. Multiple Sclerosis, 23(8):1123-1136, 2017.

[4] A. B. Mesanza, S. Lucas, A. Zubizarreta, I. Cabanes, E. Portillo, and A. RodriguezLarrad. A Machine Learning Approach to Perform Physical Activity Classification Using a Sensorized Crutch Tip. IEEE Access, 8:210023-210034, 2020.

[5] O. R. Pearson, M. E. Busse, R. W. Van Deursen, and C. M. Wiles. Quantification of walking mobility in neurological disorders. QJM - Monthly Journal of the Association of Physicians, 97(8):463-475, 2004.

[6] P. Probst, M. N. Wright, and A. L. Boulesteix. Hyperparameters and tuning strategies for random forest. Wiley Interdisciplinary Reviews: Data Mining and Knowledge Discovery, $9(3): 1-15,2019$.

[7] P. Ra and P. A. Rte. Atlas de EM, 2020.

[8] A. Souza, A. Kelleher, R. Cooper, R. A. Cooper, L. I. Iezzoni, and D. M. Collins. Multiple sclerosis and mobility-related assistive technology: Systematic review of literature. Journal of Rehabilitation Research and Development, 47(3):213-224, 2010.

[9] F. P. Thomas. Multiple Sclerosis. Pathy's Principles and Practice of Geriatric Medicine: Fifth Edition, 1(3):823-833, 2012.

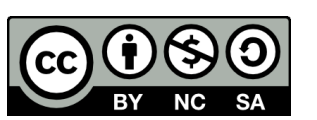

(C) 2021 by the authors. Submitted for possible open access publication under the terms and conditions of the Creative Commons Attribution CC BY-NC-SA 4.0 license (https://creativecommons.org/licenses/by-ncsa/4.0/deed.es). 